\title{
Band Assignment in Dual Band Systems: A Learning-based Approach
}

\author{
Daoud Burghal, Student Member, IEEE, Rui Wang, Student Member, IEEE, and Andreas F. Molisch Fellow, IEEE
}

\begin{abstract}
We consider the band assignment problem in dual band systems, where the base-station (BS) chooses one of the two available frequency bands (centimeter-wave and millimeterwave bands) to communicate data to the mobile station (MS). While the millimeter-wave band offers higher data rate when it is available, there is a significant probability of outage during which the communication should be carried on the centimeterwave band.

In this work, we use a machine learning framework to provide an efficient and practical solution to the band assignment problem. In particular, the BS trains a Neural Network (NN) to predict the right band assignment decision using observed channel information. We study the performance of the $\mathrm{NN}$ in two environments: (i) A stochastic channel model with correlated bands, and (ii) microcellular outdoor channels obtained by simulations with a commercial ray-tracer. For the former case, for sake of comparison we also develop a threshold based band assignment that relies on the optimal mean square error estimator of the best band. In addition, we study the performance of the NN-based solution with different NN structures and different observed parameters (position, field strength, etc.). We compare the achieved performance to linear and logistic regression based solutions as well as the threshold based solution. Under practical constraints, the learning based band assignment shows competitive or superior performance in both environments.
\end{abstract}

Index Terms-Dual Band, Neural Network, Deep Learning, band assignment.

\section{INTRODUCTION}

The large available bandwidth in the millimeter-wave (mmWave) frequency band can support the high data rates required for many applications. However, the hostile propagation conditions at high frequencies restrict the utilization of the mmWave band in cellular communication. Compared to the centimeter-wave (cmWave) band, signals in the mmWave band suffer from higher attenuation, higher diffraction loss, and are more susceptible to blockage, which reduces the reliability of the communication systems [1], [2]. To gain the advantages of both bands, next-generation wireless networks are anticipated to use both frequency bands [3], [4]. This can enhance the signal coverage, system reliability and achievable data rates.

The simultaneous usage of the two bands might not be practical due to a number of limitations at the MS side,

Part of this work was supported financially by the National Science Foundation.

The authors are at the Ming Hsieh Department of Electrical Engineering, University of Southern California, Los Angeles, CA 90089, USA (email: burghal,wang78,molisch@usc.edu). such as the limited processing capabilities, the constraint on transmission power, etc. Thus, the BS has to assign the MS to one of the two bands, in particular it has to switch the communication from the cmWave band to mmWave whenever the mmWave band is available or the other way around when the mmWave band suffers a blockage or other band propagation conditions. The band assignment (BA) problem is challenging, since the channel states in both bands might not be simultaneously observable at the BS side. In addition, using training signals over the two bands and frequent switching between the bands can be expensive and a waste of resources. To mitigate the aforementioned challenges, the BS can utilize the partial information, such as the channel state in one band, together with knowledge of BA "training data" to solve the BA problem.

Neural networks (NNs) have been successfully used in a number of wireless applications [5], [6], as they proved to learn complex relations between the input data (features) and the output values (or labels). In this work we use NNs to provide a solution to the BA problem. This is motivated by the fact that the BA problem involves complicated relations between the observed features and the BA decision; these relations can be challenging to capture analytically.

In the NN-based BA, the BS uses some of the features of the channel, such as the signal to noise ratio (SNR) in one band, the location of the MS, the signal delay, and/or the angle of departure to determine the band that could provide the highest data rate. To train the $\mathrm{NN}$, the $\mathrm{BS}$ can possibly use training data based on BA decisions at other locations and their associated observed features. Such information is relatively easy to collect as the MSs access the network regularly.

\section{A. Prior Work}

There have been a number of recent studies that considered the interplay between cmWave and mmWave bands. Refs. [2], [7], [8] utilize the angular correlation in the two bands to provide an estimate of the Angle of Arrival at mmWaves, which can be used to reduce the beamforming complexity at mmWave. Furthermore, [8] suggests using both frequency bands for data communication, and proposes a two-queue model to assign data to each band such that delay is minimized and throughput is maximized. Ref. [9] considers the downlink resource allocation in a network with a small cell BS, where the BS aims to assign the applications running on the MS's to 
the resources in the two bands. Our recent work [10] considers the band switching problem for an MS where the BS uses the observed channel states in the previous time-frames to predict the future one. Using a given channel model, the solution in [10] depends on the knowledge of the channel statistics and the path loss values, which might not be available in some practical situations. In addition, the channel states in previous time frames are usually not available during initial network access, or may convey relatively limited information for static or nomadic MSs.

Applying machine learning to solve wireless communication problems has gained considerable attention lately [5], [6]. For instance, recent studies use NNs to perform sequence detection [11], for jointly optimizing the encoding and the decoding in MIMO systems [12], and to provide power allocation in interference limited networks [13]. NNs have been also applied to learn the feature interrelation in wireless systems, Ref. [14] uses NNs to enable the BS to predict some unobserved features at the MS side, in particular, they try to infer the Angle of Arrival (AoA).

\section{B. Contribution}

Different from the above we consider the BA problem using the observed instantaneous features of the MS or its channel. Utilizing such information, the BS can reduce the required signaling, which improves the spectrum efficiency and reduces the latency in the system. The set of features available at the BS for the BA depends on the system setup, it may include: the location of the MS, the received power (or data rate) in the cmWave band 1 the delay, and the Angle of Departure (AoD) of the main multi-path component (MPC) [1].

In this work, for ease of discussion and brevity, when we assess the impact of features availability, we focus on the features in cmWave band, i.e., switching from the cmWave band to the mmWave band, as the other cases are simple extensions. Furthermore, note that switching from the mmWave band to the cmWave band based on the observed SNR value is relatively safer than the opposite direction due to the relative reliability of the cmWave band [4], [10].

We use the NN framework, considering different NN structures and optimizing over different parameters. Additionally, we study the performance of the NN-based solution over different features combinations. To evaluate the performance of the NN-based solution we consider two environments, a stochastic and a ray-tracing based environments. The stochastic environment can provide initial assessment of the behavior of the proposed solution. As in [10], we jointly generate the large scale fading in the two frequency bands. Furthermore, we utilize the mathematical tractability of the channel in this environment to develop a threshold-based BA (TBBA) scheme that we use to compare against the NN-based solution. The second environment is a data set generated by ray tracer

\footnotetext{
${ }^{1}$ We use the SNR, signal strength and rate interchangeably when we refer to one of them as a feature, since we assume that we can use one of them to calculate the others, even though that might not be correct under some circumstances (e.g., interference-dominated channels).
}

to simulate the propagation conditions in the two frequency bands on a university campus. We use this environment to verify and extend our conclusions that we made based on the stochastic environment. In both environments, we use Linear Regression (LR) and Logistic Regression (GR) based solutions as benchmarks. Note that LR and GR are basic learning technique that capture the relation between the features and the BA. In summary, the contributions of this manuscript are threefold.

- We formulate the BA problem in a machine learning framework, and consider different NN configurations to optimize the performance of the proposed approach.

- We study the impact of different features on the performance of the NN-based BA solution.

- We compare the NN-based scheme to different benchmarks. In addition to the LR-based and GR-based BA, we develop the TBBA for meaningful comparisons in the stochastic environment.

The paper is organized as follows. Sec. M model. Sec. III] introduces the NN and highlights the set of features. Sec. IV summaries the channel model, then provides the derivation of the TBBA rule, and discusses the performance of the schemes in the simulated stochastic channels and their generalization capabilities. Sec. $\mathrm{V}$ includes the description of the ray-tracing environment and the performance of the learning-based schemes in that environment. Finally, Sec. VI provides concluding remarks.

\section{System Model}

We consider a dual band cellular system, where the BS and the MS can operate in two frequency bands with center frequency $f_{b}$ and bandwidth $\omega_{b}$ in band $b \in\{c, m\}$, where $c$ and $m$ refer to the cmWave and the mmWave bands, respectively. Due to a number of practical limitations of the MS, we assume that data transmission occurs in a single frequency band at a time. The goal of the BS is to choose the band that results in the highest data rate. To focus on the basic problem, we consider a single user case, i.e., no scheduling or interference is considered; the multi-user case is left for future work.

In this work, the BA procedure depends on the scheme. In NN-based schemes, the BS feeds the observed features, denoted by set $\mathcal{F}$, to the $\mathrm{NN}$ to produce the soft decision $\tilde{\mathcal{D}}$. Then it uses $\tilde{\mathcal{D}}$ to produce the BA decision $\mathcal{D}$. Note that since we have two distinct decisions, we can assume that $\tilde{\mathcal{D}} \in$ $[0,1]$ and $\mathcal{D} \in\{0,1\}$, where " 1 " refers to an assignment to the mmWave band. Thus we can view the problem as binary classification problem. The BS uses a threshold $\gamma_{\mathrm{L}} \in[0,1]$ to map $\tilde{\mathcal{D}}$ to $\mathcal{D}$, where we assume that $\mathcal{D}=1$ when $\tilde{\mathcal{D}}>\gamma_{\mathrm{L}}$. The method we use to choose of $\gamma_{\mathrm{L}}$ is discussed in Sec. III

To train the $\mathrm{NN}$, the BS uses a data set $\mathcal{A}^{T}=$ $\left\{\mathcal{P}_{1}^{T}, \ldots, \mathcal{P}_{N_{T}}^{T}\right\}$, where the superscript $T$ denotes training, and $N_{T}$ is the number of training examples. Each example point $\mathcal{P}_{i}^{T}$ is a features-label pair $\left(\mathcal{F}_{i}, \mathcal{L}_{i}\right)$, where $\mathcal{F}_{i}$ is the set of features of the $i$ th example, and $\mathcal{L}_{i} \in\{0,1\}$ is the true label of that example, where " 1 " refers to the case when the data 


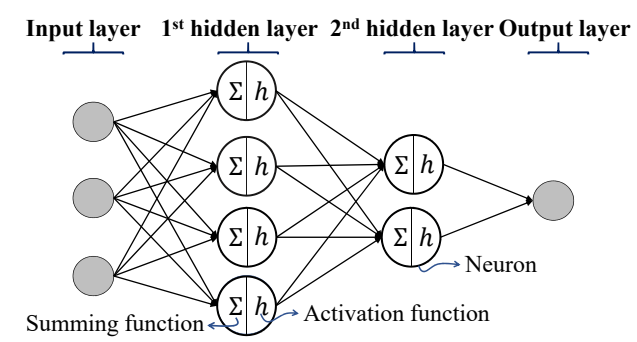

Fig. 1. Illustration of a neural network with two hidden layers.

rate in the mmWave band is larger than the data rate in the cmWave band. We assume that $\mathcal{A}^{T}$ is available to the BS, for instance through previous decisions or an initial network training phase. However, the procedure to acquire $\mathcal{A}^{T}$ is out of the scope of the paper.

For clarity, we defer the discussion of the BA assignment procedure using the TBBA to subsection IV-A.

\section{Neural Network AND FeAtures}

\section{A. Neural Networks}

Artificial neural networks have been successfully applied to many complex practical problems. An NN consists of one or more layers, each of which has a number of parallel neurons (nodes), see Fig. 1. The neuron performs a weighted combination of the input features and then passes it through a possibly non-linear transformation, also known as an activation function, e.g., a sigmoid function. The weights are determined during the training phase over a training set $\mathcal{A}^{T}$. where the goal is to minimize the prediction error of the label values at the output of the $\mathrm{NN}$ over the observed data points.

In this work we use feedforward NNs, where the output $\mathcal{D}$ depends solely on $\mathcal{F}$, i.e., the output at one instance does not influence the output at other time instances. To optimize the performance, we consider different NN structures. Note that while a large number of layers allows the NN to learn "difficult" aspects of the problem, this also increases the chances of over-fitting. In the simulation, we use several NNs structures with up to four hidden layers and up to 100 nodes in total. To guard against over-fitting we use L2 norm regularization in the training phase with different regularization coefficients $\alpha$ [15].

\section{B. Features}

In this work we consider upto five features, i.e., channel characteristics that are used as input: $\left(f_{1}\right)$ the distance from the BS to the MS $d$ in meters, $\left(f_{2}\right)$ the angular position of the MS $\theta$ in $\mathrm{rad},\left(\mathrm{f}_{3}\right)$ the received signal strength or the SNR in the cmWave band in $\mathrm{dBm}$ or $\mathrm{dB}$ (or equivalently the data rate in this paper), $\left(\mathrm{f}_{4}\right)$ the delay of the main MPC in seconds, and $\left(\mathrm{f}_{5}\right)$ the power of the main MPC in $\mathrm{dBm}$, note that we refer to the MPC with highest power as the main MPC.

Note that $\left(f_{1}\right)$ and $\left(f_{2}\right)$, i.e., $(d, \theta)$, represent the polar coordinates of the MS with respect to the $\mathrm{BS}$, which may be estimated by localization techniques such as GPS. To extract $\left(f_{4}\right)$ large bandwidth might be required, for $\left(f_{5}\right)$ the use of antenna array is necessary. For $\left(f_{3}\right)$, observation of the small-scale-averaged power are sufficient, because small-scale fading is usually averaged out by frequency diversity in largebandwidth systems. This power can be observed at either the BS or the MS. As a result, the available features depend on the system implementations. In this work we consider several combinations of the above features.

As typically done in machine learning, we perform preprocessing to the features, such as normalizing the input features. We also use logarithmic scale for distances and power, as this may linearize their relation with one another.

\section{Training and Testing}

Let the set $\mathcal{A}$ denotes the entire data set we use in each environment, where each point $\mathcal{P}_{i} \in \mathcal{A}$ represents the features label pair $\left(\mathcal{F}_{i}, \mathcal{L}_{i}\right)$, where $i \in\{1, \ldots, N\}$ and $N=|\mathcal{A}|$, |.| denoting the cardinality operator. In the simulation we randomly split $\mathcal{A}$ into a training set $\mathcal{A}^{T}$ and a testing set $\mathcal{A}^{S}$, where $\mathcal{A}=\mathcal{A}^{T} \cup \mathcal{A}^{S}$ and $\mathcal{A}^{T} \cap \mathcal{A}^{S}=\emptyset$. We further split $\mathcal{A}^{T}$ into a training subset $\mathcal{A}_{t}^{T}$ and a validation subset $\mathcal{A}_{v}^{T}$, $\mathcal{A}_{t}^{T} \cap \mathcal{A}_{v}^{T}=\emptyset$. More details about this are provided in the next sections. During the training phase, as it is commonly used in binary classification problems, the performance of the $\mathrm{NN}$ is evaluated using Cross Entropy (CE) cost function, i.e.,

$$
\overline{\mathcal{E}}_{\mathrm{CE}, X}=\frac{1}{N_{X}} \sum_{i}^{N_{X}}\left(-\mathcal{L}_{i} \log \left(\tilde{\mathcal{D}}_{i}\right)-\left(1-\mathcal{L}_{i}\right) \log \left(1-\tilde{\mathcal{D}}_{i}\right)\right) .
$$

where the subscript $X \in\{T, V\}$ is used to identify, respectively, the training and the validation. We use Monte-Carlo cross validation to improve our estimate of the validation error, in which we repeat the random split of $\mathcal{A}^{T}$ to $\mathcal{A}_{t}^{T}$ and $\mathcal{A}_{v}^{T}$, and rerun the training and the validation. Then we choose the network structure and regularization coefficient that achieve the smallest average $\overline{\mathcal{E}}_{\mathrm{CE}, \mathrm{V}}$. In this paper, we focus on the average number of BA errors, i.e., we use

$$
\overline{\mathcal{E}}_{X}=\frac{1}{N_{X}} \sum_{i}^{N_{X}}\left|\mathcal{D}_{i}-\mathcal{L}_{i}\right|
$$

where the subscript $X \in\{S, V\}$ denotes, respectively, the testing and the validation. To choose $\gamma_{\mathrm{L}}$ we use the value in $[0,1]$ that results on the smallest average $\overline{\mathcal{E}}_{V}{ }^{2}$

Finally note that we use similar training, cross validation and method to obtain the hard decisions for LR-based and GR-based techniques as well.

\section{Stochastic EnVIROnMENT}

Here we study the performance of the NN-based solution in stochastically generated channels. This will provide an initial assessment of the performance of the NN-based scheme with various feature combinations.

\footnotetext{
${ }^{2}$ One may choose different performance metric, e.g., probability of error, and/or other methods to select $\gamma_{\mathrm{L}}$, such as the area under ROC curve [15], however we chose the above for clarity and ease of discussion.
} 


\section{A. Channel Model and the TBBA Scheme}

Similar to [10], we assume the channel model consists of the pathloss and the large-scale fading. For band $b \in\{c, m\}$, the SNR on a logarithmic scale $(\mathrm{dB})$ is given by

$$
\mathrm{SNR}^{b}=P_{\mathrm{tx}}^{b}-P L^{b}-N_{0}^{b}+S^{b},
$$

where $P_{\mathrm{tx}}^{b}$ is the transmitted power, $P L^{b}$ is the path loss, $N_{0}^{b}$ is the noise level, and $S^{b}$ is a random process that represents the large-scale fading.

With the assumption that the shadowing values in the two bands are jointly normal (on a logarithmic scale), the BS can use the observed channel state or rate in the cmWave band to infer their values in the mmWave band. Then, ideally it would assign the MS to the band with the largest rate. Since the channel state in the mmWave band is not observable, we may assign the MS to the mmWave band, if the probability to achieve a larger rate in the mmWave band is greater than $\gamma_{T} \in[0,1]$, i.e.,

$$
\mathbb{P}\left(\mathbf{R}^{m} \geq \mathbf{R}^{c} \mid \mathbf{R}^{c}=r_{c}\right) \geq \gamma_{T},
$$

where $\mathbf{R}^{b}$ is the rate in band $b$. Note that the left hand side of (3) is equivalent to the optimum mean square error estimator of the BA decision for given channel state in the cmWave band. Using the Shannon capacity equation, the rate in band $b$ is

$$
\mathbf{R}^{b}=\omega_{b} \log \left(1+\gamma_{b}^{\prime} 10^{\gamma^{\prime \prime} S^{b}}\right) .
$$

where $\gamma^{\prime \prime}=0.1$ and $\gamma_{b}^{\prime}=10^{\left(P_{\mathrm{tx}}^{b}-P L^{b}-N_{0}^{b}\right) \times 0.1}$. Using (4) we can rewrite the probability in 3 as:

$$
\mathbb{P}\left(\omega_{m} \log \left(1+\gamma_{m}^{\prime} 10^{\gamma^{\prime \prime} S^{m}}\right) \geq r_{c} \mid \omega_{c} \log \left(1+\gamma_{c}^{\prime} 10^{\gamma^{\prime \prime} S^{c}}\right)=r_{c}\right)
$$$$
=\mathbb{P}\left(S^{m} \geq v_{1} \mid S^{c}=v_{0}\right),
$$

where $v_{0}=\frac{1}{\gamma^{\prime \prime}} \log _{10}\left(\frac{1}{\gamma_{c}^{\prime}}\left(\exp ^{r_{c} / \omega_{c}}-1\right)\right)$ and $v_{1}=$ $\frac{1}{\gamma^{\prime \prime}} \log _{10}\left(\frac{1}{\gamma^{\prime}}\left(\exp ^{r_{c} / \omega_{m}}-1\right)\right)$. With the assumption that $S^{m}$ and $S^{c}$ are jointly normal, it is enough to determine the conditional mean $\mu_{m \mid c}$ and variance $\sigma_{m \mid c}^{2}$ to calculate the probability in (5), which can be shown to be

$$
\mu_{m \mid c}=\rho_{m, c} \frac{\sigma_{m}}{\sigma_{c}} v_{0} \quad \text { and } \quad \sigma_{m \mid c}^{2}=\left(1-\rho_{m, c}^{2}\right) \sigma_{m}^{2},
$$

where $\rho_{m, c}$ is the correlation coefficient of $S^{m}$ and $S^{c}$. Thus we have

$$
\mathbb{P}\left(S^{m} \geq v_{1} \mid S^{c}=v_{0}\right)=\mathbb{Q}\left(\frac{v_{1}-\mu_{m \mid c}}{\sigma_{m \mid c}}\right) \geq \gamma_{T},
$$

where $\mathbb{Q}($.$) is the Q-function [16]. Taking the inverse of Q-$ function, and rearranging the terms, we have

$$
v_{1} \leq \mathbb{Q}^{-1}\left(\gamma_{T}\right) \sigma_{m \mid c}+\mu_{m \mid c}=\mathbb{Q}^{-1}\left(\gamma_{T}\right) \sigma_{m \mid c}+\rho_{m, c} \frac{\sigma_{m}}{\sigma_{c}} v_{0} .
$$

Solving for $v_{0}$, then the BS would assign the MS to the mmWave band if the following condition is satisfied:

$$
S^{c} \geq \frac{\sigma_{c}}{\rho_{m, c} \sigma_{m}}\left(v_{1}-\mathbb{Q}^{-1}\left(\gamma_{T}\right) \sigma_{m \mid c}\right),
$$

which is a function of the observed rate $\gamma_{T}, r_{c}, v_{1}$ and the statistics of the environment. Here we set $\gamma_{T}=0.5$ as it is the "natural" choice in this case, we provide further discussion about that in [17]. To calculate $v_{1}$, we need to calculate $\gamma_{m}^{\prime}$, which in turn requires the knowledge of the pathloss.

\section{B. The Data Set}

To generate the channel realizations in the two bands, we use the model suggested in [10], with shadowing standard deviation $\sigma_{b}$, decorrelation distance $d_{\mathrm{dcor}}^{b}$ in band $b$, and a correlation coefficient $\rho_{c, m}$. We further assume that the pathloss follows a break point pathloss model [1], with a break distance $d_{\text {break }}$ and a propagation exponent 2 for $d \leq d_{\text {break }}$ and $\epsilon$ for $d>d_{\text {break }}$. Table [ summarizes the values used for generating the data set.

We assume that the BS is located at the center of a square cell with a side length of $500 \mathrm{~m}$, the data set consists of 2000 data points, which correspond to 2000 uniformly distributed MSs around the BS. In this data set, to simplify the simulation environment, we focus on three features: location of the MS $(d, \theta)$ and the power in the cmWave band.

In this environment, we use $65 \%$ of the data set for training. For the Monte-Carlo cross validation, we take around $20 \%$ of $\mathcal{A}^{T}$ for the validation subset $\mathcal{A}_{v}^{T}$. We generate 1000 independent channel/cell realizations to assess the performance of the learning based BA and the TBBA in the stochastic environment.

\section{Performance}

We can view each of the 1000 cell realizations as a different cell, which is reasonable in cellular system. Then for every realization we repeat the training, validation and then testing.

Table-[ summarizes the results for all seven feature combinations. The 5th, the 6th and the 7th rows show the test errors of the NN-based, the GR-based and the LR-based solutions, respectively. We emphasize that the displayed performance values by no means the optimal values, as we have considered a limited number of structures and parameters and performed a grid search over them. The last row in the table shows the performance of the TBBA. In the generated data set we have about $50.7 \%$ of the labels are " 0 ". As a result, assigning the cmWave band for all points would result in error equal 0.493, we use this value as a reference for comparison.

In general, we notice the learning techniques provide significant improvements over the cmWave-only BA, with an advantage to the NN-based scheme over the other schemes, as the $\mathrm{NN}$ is able to learn the non-linearity in the feature(s)/BA mapping. This can be observed in the performance for the first features combination (c-1), i.e., the location of the MS, where we notice that the NN-based BA outperforms the other learning approaches. Next, adding the received power in the cmWave band, (c-2), provides an evident performance gain for all learning approaches. In fact, it seems that any other combination with the power information would provide comparable performance, especially when we use the angle information as in (c-3). 


\begin{tabular}{|c|c|}
\hline Variable & Band c/m \\
\hline $\boldsymbol{f}_{\boldsymbol{b}}$ & $2.5 / 28 \mathrm{GHz}$ \\
\hline Bandwidth $\omega_{\mathbf{b}}$ & $10 / 100 \mathrm{MHz}$ \\
\hline $\boldsymbol{P}_{\mathbf{t x}}^{b}$ & $15 / 22 \mathrm{dBm}$ \\
\hline $\boldsymbol{\epsilon}$ & 4 \\
\hline $\boldsymbol{d}_{\text {break }}$ & $50 \mathrm{~m}$ \\
\hline $\boldsymbol{d}_{\text {dcor }}$ & $25 / 24$ \\
\hline $\boldsymbol{\sigma}_{\boldsymbol{b}}$ & $5 / 7 \mathrm{~dB}$ \\
\hline $\boldsymbol{\rho}_{\boldsymbol{m}, \boldsymbol{c}}$ & 0.75 \\
\hline Noise Spectral Density & $-174 \mathrm{dBm} / \mathrm{Hz}$ \\
\hline
\end{tabular}

TABLE I

STOCHASTIC CHANNEL SIMULATION CONFIGURATIONS

To analyze the improvements in (c-2), (c-3) and (c-4) compared to (c-1), we study the performance using the distanceonly feature in (c-5), power-only feature in (c-6) and angleonly feature in (c-7). While the bad performance in (c-7) is somewhat expected, as the received power is independent of angle on average, the power in the cmWave band seems to reveal more information about the BA than the distance. In fact, we notice that the performance in (c-4) is close to (c-6). This should not be surprising, as the shadowing is better captured with the received power in the cmWave band compared to the distance. In the light of this observation, we notice an improvement in (c-3) compared to (c-4), where the angle will provide an additional information to the power where we can identify cluster of similar BA decisions. In general, the performances of the NN-based scheme and both regression schemes are comparable in (c-5) and (c-6), indicating that a simple linear function could be used for BA in these two cases, which could be justified by the fact that in (c-5) the average power could be approximated as a linear function of distance, while for (c-6) with the assumption of jointly normal channels, the linear function is an optimal estimator.

For the TBBA, Table III shows that the TBBA provides an improvement compared to the cmWave-only BA. We notice that using combination (c-2), (c-3), (c-4) and (c-6), the learning based schemes can achieve similar performance to the TBBA, these have been achieved without providing the structure and the statistics of the channels. In fact, the NN is able to outperform the TBBA in (c-2) and (c-3), interestingly, with power only feature the learning based solutions are roughly as good as the TBBA.

\section{Generalization}

As an alternative to the method above, we might want to train the learning based approaches on a few cell realizations and apply it on other realizations, i.e., we are interested in transfer the learning experience without the need to go through the training phase again for each cell. In general, learning in stochastic environments is difficult, as the randomness increases the unpredictability of the labels and complicates the description of the mapping (if it exists) between the features and the labels. As a result, we expect a degradation in the performance as we try to generalize the learned relations.

We use the same data set to study the generalizations of the solutions. However, we divided the 1000 cell realizations into group of 50 realizations. For each group, we use 30 realizations for training, five for validation and 15 for testing. We average the performance for the 20 groups. We highlight here the results for three cases: for (c-1) we have $\overline{\mathcal{E}}_{S}$ for NN, GR and LR respectively: $\{.27, .271, .271\}$, for (c-2) we have $\{.195, .195, .198\}$, and for (c-6) we have $\{.195, .196, .196\}$, and the TBBA has a test error value .1953 We notice a degradation in the performance in (c-1) and (c-2), as the learning approaches are not able to use some of the learned relations, that were deduced from the correlation between shadowing in the training cell realizations, for the BA over the test data set, as the latter is independent of the training data set in this case. Interestingly, we notice that for (c-6), the learning based approaches provide similar performance as the TBBA, which indicates that they are good alternative to the TBBA for some feature combinations.

Finally, we conclude this section by emphasizing three limitations of the TBBA; first, it requires the knowledge of the exact pathloss to calculate $v_{1}$. Second, the correlation models and the shadowing distribution should be known to the BS. Third, as we noticed in IV-C since the TBBA was derived based on an average metric, it does not exploit the available information in the given realization of the environment.

\section{Simulated Campus Environment}

\section{A. The Data Set}

To assess the performance in a more realistic setting, we simulate the propagation channel in a campus environment by means of a commercial ray-tracing tool, Wireless InSite [18]. The input to the ray-tracer includes the 3D models of the buildings and the electromagnetic characteristics of the building materials as well as models of foliage. Wireless InSite performs ray launching, emitting rays (representing plane waves) from the transmitter into all directions, and following each ray as it interacts (reflection, diffraction, transmission) with the objects in the environment. The output is a list of parameter vectors that contains the power, propagation delay, the AoD and AoA, for each MPC. Simulation results have been compared to measurements in a variety of settings and shown to provide good agreement [18]. This simulation has been conducted based on the model of University Park Campus, University of Southern California (USC), which is shown in Fig.2-(a). The detailed simulation configurations are

\footnotetext{
${ }^{3}$ Note that the test error is different here compared to one that is shown in Table II as the test data sets are different in the two cases.
} 
listed in Table III Simulation results in the same environment are used in [19], [20].

The data set has about 1150 points, i.e., $|\mathcal{A}|=1150$, each point contains all the five features. The label that is associated with each point is whether the rate in the mmWave band is larger than the one in the cmWave band. To calculate the rate we use the Shannon capacity with bandwidth and noise spectral density that are shown in Table III

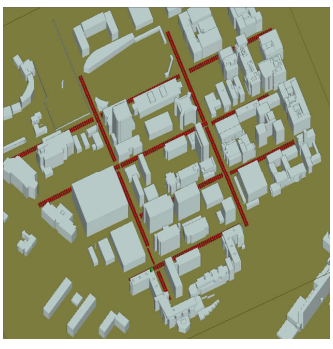

(a)

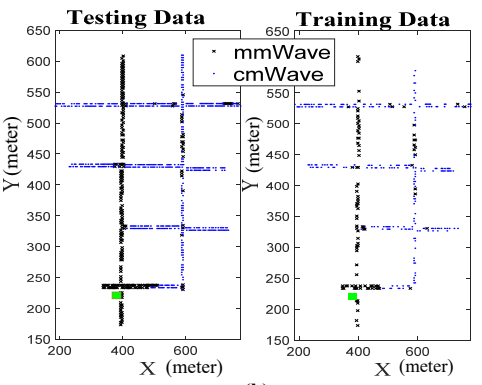

(b)
Fig. 2. (a) Ray-tracing simulation environment. The green dot is the BS located above the rooftop, while simulated MSs are red routes. Gray objects represent the buildings. The light/dark green 3D polygons denote foliage features with different trees density. (b) Using $70 \%$ of the data for testing, from left to right $\mathcal{A}^{S}$ and $\mathcal{A}^{T}$ with the corresponding labels.

Since acquiring a large number of data points may not be practical for the $\mathrm{BS}$, using a large portion of the data set for training may produce misleading results. Here we use only $30 \%$ of $\mathcal{A}$ for training. To apply the Monte-Carlo cross-validation method, we randomly choose $80 \%$ of $\mathcal{A}^{T}$ for training and $20 \%$ for validation. The network is then tested on $\mathcal{A}^{S}$, i.e., the remaining $70 \%$ of the data set. Fig. 2-(b) shows an example of the sets $\mathcal{A}^{S}$ and $\mathcal{A}^{T}$.

\section{B. Performance}

We first point out that in this environment using the cmWave band only would result in an error equal to 0.3 , i.e., the percentage of " 1 " in $\mathcal{A}$ is $30 \%$. Table-IV summarizes the results of the solutions. The last three rows show the performance of learning-based schemes when simulated over the described training set. The the 7th row shows the number of layer (for an NN that consists of 100 nodes), the regularization coefficient $\alpha$ and the chosen threshold value $\gamma_{\mathrm{L}}{ }^{4}$

Combinations (c-1) and (c-8) show the cases when we use the location or the delay and AoD, these two are usually related as several localization techniques use the delay and AoD to determine the location. The performance in the two cases are comparable, even though we may not have Line of Sight (LOS) in all the cases. We also note that the NN-based approach significantly outperforms the other two approaches.

Adding the power to the two combinations above, as in (c2) and (c-7), improves the performance similar to the previous section, especially for both regression-based BA. In fact, in (c-7) their performance are slightly higher than the error

\footnotetext{
${ }^{4}$ Again, we do not claim that the shown structures are the optimal choices.
}

for NN-based BA. The performance gain in (c-2) and (c7) can be partially explained by the good results in (c-5) that uses the cmWave power only. For comparison, a scheme that only exploits the distance feature (c-6) shows relatively poor performance for all the learning based schemes. This is consistent with our findings in the previous section, as the shadowing and the blockage have major impact on the quality of wireless channels especially in the mmWave band. Similar comparisons can be done with a delay-only (not shown in the table) scheme, which provides an improvement compared to distance only, with $\overline{\mathcal{E}}_{\mathrm{S}}=0.17$ for NN-based, $\overline{\mathcal{E}}_{\mathrm{S}}=0.165$ for GR-based and $\overline{\mathcal{E}}_{\mathrm{S}}=0.166$ for the LR-based BA. This performance could be expected in that delay may reflect a more realistic "effective" distance, note that non-LOS links will show a longer delay even if they have similar geographic distance as their LOS counterpart. A combination of delay and distance with power, in (c-3) and (c-4), shows small improvement over power-only, however, they show significant improvement over distance-only and delay-only cases.

Finally, we notice that in this environment, the performance gaps between the NN based and other learning-based BA solutions are in general larger than for the previous one. Which suggests that in a more realistic environment, the NN is especially useful.

\section{CONCLUSION}

In dual-band systems, where the BS and the MS can communicate in either the cmWave or mmWave frequency bands, the BS should assign the MS to the appropriate band. In this paper we explore learning based approaches to provide a solution to the band assignment problem. We consider two environments to assess the performance of the proposed techniques and gain insight about the impact of different features: ray-tracing and stochastic environments.

The performance of the schemes depends on the set of the available features. In both environments, the learning based approaches show impressive performance when the SNR in one band is known. In general, NNs (with relatively small number of nodes and hidden layers) show good performance using features that are relatively easy to acquire, such as the signal strength in one band and the delay of the main path. This indicates the practicality of the learning based schemes. The results also point out the importance of power correlation when studying and analyzing dual-band systems.

\section{REFERENCES}

[1] A. F. Molisch, Wireless Communications. IEEE Press - Wiley; 2 edition, 2011

[2] A. Ali, N. González-Prelcic, and R. W. Heath, "Estimating millimeter wave channels using out-of-band measurements," in Info. Theory and App. Works, (ITA), 2016. IEEE, 2016, pp. 1-6.

[3] J. G. a. Andrews et al., "What will 5g be?" IEEE Jour. on select. areas in comm., vol. 32, no. 6, pp. 1065-1082, 2014.

[4] N. González-Prelcic et al., "Millimeter-wave communication with outof-band information," IEEE Comm. Mag., vol. 55, no. 12, pp. 140-146, 2017.

[5] M. Chen et al., "Machine learning for wireless networks with artificial intelligence: A tutorial on neural networks," arXiv preprint arXiv:1710.02913, 2017. 


\begin{tabular}{|c|c|c|c|c|c|c|c|c|c|c|}
\hline Variable & Band c/m & Feature /Combination & c-1 & c-2 & c-3 & c-4 & c-5 & c-6 & c-7 & c-8 \\
\hline $\mathbf{f}_{\mathrm{b}}$ & $2.5 / 28 \mathrm{GHz}$ & $d$ & $\checkmark$ & $\checkmark$ & $\checkmark$ & & & $\checkmark$ & & \\
\hline Ant. Pattern & Isotropic & $\theta$ & $\checkmark$ & $\checkmark$ & & & & & & \\
\hline Ant. Polarization & Vertical & cmWave Power & & $\checkmark$ & $\checkmark$ & $\checkmark$ & $\checkmark$ & & $\checkmark$ & \\
\hline $\mathbf{P}_{\mathrm{tx}}^{\mathrm{b}}$ & $15 / 30 \mathrm{dBm}$ & Delay & & & & $\checkmark$ & & & $\checkmark$ & $\checkmark$ \\
\hline BS height & $45 \mathrm{~m}$ & AoD & & & & & & & $\checkmark$ & $\checkmark$ \\
\hline MS height & $2 \mathrm{~m}$ & Numb Layers/ $\alpha / \gamma_{L}$ & $2 / .15 / .45$ & $4 / .15 / .55$ & $1 / .05 / .5$ & $1 / .1 / .6$ & $2 / .1 / .35$ & $3 / .5 / .45$ & $4 / .3 / .6$ & $3 / .5 / .55$ \\
\hline Max. Diffraction & $2 / 1$ & $\mathbf{N N} \mathcal{E}_{\mathbf{S}}$ & .078 & .061 & .072 & .074 & .085 & .182 & .067 & .093 \\
\hline Max. Reflection & 10 & $\operatorname{GR} \mathcal{E}_{\mathrm{S}}$ & .178 & .062 & .082 & .081 & .083 & .183 & .082 & .182 \\
\hline & & $\operatorname{LR} \overline{\mathcal{E}}_{\mathrm{S}}$ & .176 & .078 & .088 & .078 & .081 & .178 & .072 & .188 \\
\hline
\end{tabular}

TABLE III

RAY-TRACING SIMULATION

CONFIGURATIONS OF USC

CAMPUS
TABLE IV

PERFORMANCE OF THE LEARNING TECHNIQUES ON RAY-TRACING DATA, UNDER DIFFERENT FEATURE AVAILABILITY, NOTE THAT THE PERCENTAGE OF POINTS WITH LABELS EQUAL TO "1" IS APPROXIMATELY 30\%.

[6] T. OShea and J. Hoydis, "An introduction to deep learning for the physical layer," IEEE Trans. on Cogn. Comm. and Net., vol. 3, no. 4, pp. 563-575, 2017.

[7] T. Nitsche et al., "Steering with eyes closed: Mm-wave beam steering without in-band measurement," in 2015 IEEE Conf. on Comp. Comm. (INFOCOM), April 2015, pp. 2416-2424.

[8] M. Hashemi et al., "Out-of-band millimeter wave beamforming and communications to achieve low latency and high energy efficiency in 5g systems," IEEE Trans. on Comm., 2017.

[9] O. Semiari, W. Saad, and M. Bennis, "Joint millimeter wave and microwave resources allocation in cellular networks with dual-mode base stations," IEEE Trans. on Wirel. Comm., 2017.

[10] D. Burghal and A. F. Molisch, "Rate and outage probability in dual band systems with prediction-based band switching," IEEE Wireless Communications Letters, pp. 1-1, 2018.

[11] N. Farsad and A. Goldsmith, "Neural network detection of data sequences in communication systems," arXiv preprint arXiv:1802.02046, 2018.

[12] T. J. a. O'Shea et al., "Deep learning based mimo communications," arXiv preprint arXiv:1707.07980, 2017.

[13] H. Sun, X. Chen, Q. Shi, M. Hong, X. Fu, and N. D. Sidiropoulos, "Learning to optimize: Training deep neural networks for wireless resource management," arXiv preprint arXiv:1705.09412, 2017.

[14] S. Navabi, C. Wang, O. Y. Bursalioglu, and H. Papadopoulos, "Predicting wireless channel features using neural networks," in Comm. (ICC), 2016 IEEE Int Conf. on. IEEE, 2018, pp. 1-6.

[15] I. Goodfellow et al., Deep Learning. MIT Press, 2016.

[16] M. Abramowitz and I. A. Stegun, Handbook of mathematical functions: with formulas, graphs, and mathematical tables. Courier Corporation, 1964, vol. 55.

[17] D. Burghal, R. Wang, and A. F. Molisch, "Deep learning and Gaussian process based solutions to band assignment in dual band systems," IEEE Trans. on Wirel. Comm., p. To be sumbitted, 2018.

[18] W. I. Remcom, "https://www.remcom.com/wireless-insite-empropagation-softwareinsite-wireless-em-propagation-more-info," online, accessed: March 2017.

[19] A. Adhikary, et al., "Joint spatial division and multiplexing for mmwave channels," IEEE Jour. on Selec. Areas in Comm., vol. 32, no. 6, pp. 1239-1255, June 2014.

[20] Z. Li et al., "Joint optimization of hybrid beamforming for multi-user massive mimo downlink," IEEE Trans. on Wirel. Comm., pp. 1-1, 2018. 